\title{
Leaders
}

\section{Leptin and exercise: new directions}

Initial information on the newly discovered hormone leptin suggests a primary role in energy balance and body weight maintenance. Recent published information suggests that leptin has an impact on several physiological systems, including neuroendocrine and immune function, as well as being involved in growth and development. Although the role of leptin in these areas is only partially understood at best, even less is known about the effect of exercise on plasma leptin concentrations. Further, if exercise has an impact on leptin concentration, how then does exercise affect overall leptin function? This article considers leptin function and the impact that exercise has on blood leptin concentrations, and suggests future directions for research on exercise and leptin.

Leptin, a hormone synthesized primarily by adipose tissue and secreted into the circulatory system, is a purported satiety factor with receptors in the hypothalamus. Human leptin is a relatively small protein $(16 \mathrm{kDa})$ which shares a high degree of homology with other species such as mice $(84 \%)$ and rats $(83 \%)$. Although rare in humans, leptin mutations in mice result in leptin deficiency and lead to early onset obesity, hyperphagia, and hypothalamic hypogonadism. Unlike leptin deficient mice, humans deficient in leptin do not suffer from hyperinsulinaemia, hyperglycaemia, hypercorticism, or hypothermia. Although regulation of leptin synthesis and release is poorly understood, a distinct circadian pattern for plasma leptin concentrations has been observed and is similar to that of prolactin and thyroid stimulating hormone. ${ }^{1}$ Conflicting evidence implicates insulin as a stimulator for leptin release and/or synthesis; however, plasma leptin concentrations may change independently of plasma insulin change. To date, two interventions consistently lower plasma leptin concentrations: dietary energy restriction and a single exercise session. In contrast, tumour necrosis factor $\alpha$, which is present during immune response to foreign bodies, stimulates leptin release into the circulation.

Although many different factors are involved in leptin regulation, recent evidence suggests that it is stored in vesicles within adipose tissue, and this may complicate any association between plasma leptin concentrations and leptin synthesis regulation. For example, $\beta-3$ receptor blockade in adipose tissue decreases leptin mRNA concentrations in adipose tissue when compared with nonblocked control groups, despite unaltered plasma leptin concentrations. ${ }^{2}$ Interestingly, plasma leptin concentrations are consistently associated with adiposity in a negative feed back loop (possibly to regulate food intake).

Leptin acts through a membrane bound receptor (Ob-R) identified in hypothalamic and haemopoietic cells. A homodimer, the $\mathrm{Ob}-\mathrm{R}$ receptor is a member of the class I cytokine receptor family which includes interleukin-6. In the hypothalamus, leptin activated $\mathrm{Ob}-\mathrm{R}$ receptors are thought to regulate eating behaviour, cortisol release, immune function, and other growth factors-for example, elevated leptin concentration signals adequate nutrition and permits growth and sexual development while maintaining eating behaviour. In haemopoietic cells, however, activated leptin receptors stimulate platelet aggregation possibly leading to accelerated vascular disease. ${ }^{3} \mathrm{Ob}-\mathrm{R}$ mutations are rare in humans, but lead to the same disorders as found in leptin deficient patients.

Studies examining the effects of a single exercise session and plasma leptin have found conflicting results. Dirlewanger $e t a l^{4}$ found no changes in plasma leptin in response to moderate exercise performed during a three day period. Energy intake was kept isoenergetic at either 1.3 times basal metabolic rate or increased to meet the energy expenditure induced by the exercise. Perusse et $a \bar{l}$ measured plasma leptin before, after 10-12 minutes of 50 W cycle ergometry, and immediately after reaching maximal exertion. No differences in plasma leptin were found when compared with the baseline value.

In contrast, Essig et $a l^{6}$ found a $30 \%$ reduction in leptin 48 hours after exercise that required about $6270 \mathrm{~kJ}(1500$ kcal) of energy expenditure. Using the same subjects and experimental design, no differences were found at any time points after an exercise session requiring $3344 \mathrm{~kJ}$ ( $800 \mathrm{kcal})$ of energy expenditure. Tuominen et al also found a $34 \%$ decrease in serum leptin 44 hours after a two hour exercise period performed at $75 \% \dot{\mathrm{V}}_{2} \mathrm{MAX}$. Hilton and Loucks ${ }^{8}$ examined calorie restriction, exercise, and exercise with calorie restriction. Exercise with calorie restriction was the only treatment that produced a decrease in plasma leptin concentrations 24 hours after exercise. Thong et al found somewhat similar results by inducing weight loss through diet restriction only, exercise only, or exercise without weight loss. The diet only and exercise only protocols both resulted in significant reductions in body weight $(7.5 \mathrm{~kg})$ and in plasma leptin (about $5 \mathrm{ng} / \mathrm{ml}$ ), yet the exercise without weight loss intervention produced no change in plasma leptin concentration.

Longitudinal exercise training studies have also reported conflicting results. Kraemer et $a l^{10}$ did not find any changes in leptin in obese women after a nine week aerobics class (estimated energy expended was $1256 \mathrm{~kJ}$ per session). Subjects maintained their normal diets throughout the study. Even though physical fitness improved, subject energy expenditure in conjunction with no reduction in energy intake may not have been enough of a stimulus to reduce plasma leptin. Conversely, Gutin et $a l^{11}$ found decreased plasma leptin in overweight children participating in a four month structured exercise and play programme.

Although existing published information is inconclusive, the results from studies evaluating a single exercise session indicate that leptin concentrations can be reduced in the days after exercise if the exercise session meets an energy expenditure threshold. Results from longitudinal exercise training studies are less clear. If plasma leptin concentrations are to be altered, an undefined threshold for total energy deficit as a result of either exercise training or reduced calorie intake probably exists.

Considering that obesity is a primary health concern and that many people who loose weight regain part if not all of that weight and that leptin in animal models is involved in regulating eating behaviour, understanding the impact of various lifestyle interventions such as exercise on plasma leptin concentrations and the regulation of leptin release and/or synthesis is an important public health concern. 
Future exercise research studies should focus on the following areas.

(1) Are adverse health consequences associated with elevated plasma leptin concentrations? If so, are the adverse consequences a direct result of leptin concentrations or the result of adiposity or lifestyle behaviour?

(2) What are the benefits of lowering plasma leptin concentrations through exercise and/or diet if they are involved in the negative feedback loop regulating eating behaviour?

(3) Does a single exercise session alter plasma leptin concentrations directly or are altered plasma leptin concentrations a result of a change in the balance of energy intake and expenditure? Currently, the evidence suggests that the energy balance is more important. However, positive energy balance states have not been tested with or without exercise.

(4) What is the mechanism(s) for exercise altered regulation of leptin synthesis and release?

(5) What impact does both a single exercise session and habitual exercise participation have on leptin synthesis and/or release, and how does an altered plasma leptin concentration impact on leptin receptor density (Ob-R receptors in the hypothalamus)?

In conclusion, leptin is known to be involved in physical and sexual maturity; however, we do not know whether elevated leptin concentration is a symptom or underlying factor in obesity, nor do we understand how exercise regulates plasma leptin concentrations.
J L DURSTINE R W THOMPSON K L DROWATZKY W P BARTOLI

Department of Exercise Science

University of South Carolina

Columbia, SC 29208, USA

ldurstine@sph.sc.edu

1 Sinha MK, Ohannesian JP, Heiman ML, et al. Nocturnal rise of leptin in lean, obese, and non-insulin-dependent diabetes mellitus subjects. $f$ Clin Invest 1996;97:1344-7.

2 Bramlett SB, Zhou J, Harris RBS, et al. Does beta-3-adrenoceptor blockade attenuate acute exercise-induced reductions in leptin mRNA? f Appl Physiol 1999;87:1678-83.

3 Nakata M, Yada T, Soejima N, I Maruyama. Leptin promotes aggregation of human platelets via the long form of its receptor. Diabetes 1999;48:426-9.

4 Dirlewanger M, Di Vetta V, Giusti P, et al. Effect of moderate physical activity on plasma leptin concentration in humans. Eur F Appl Physiol 1999;79: 331-5.

5 Perusse L, Collier G, Gagnon J, et al. Acute and chronic effects of exercise on leptin levels in humans. F Appl Physiol 1997;83:5-10.

6 Essig DA, Alderson NL, Ferguson MA, et al. Delayed effects of exercise on the plasma leptin concentration. Metabolism 2000;49:395-9.

7 Tuominen JA, Ebeling P, Laquier FW, et al. Serum leptin concentration and fuel homeostasis in healthy man. Eur f Clin Invest 1997;27:206-11.

8 Hilton LK, Loucks AB. Low energy availability, not exercise stress, supresses the diunal rhythm of leptin in healthy young women. Am F Physiol Endocrinol Metab 2000;278:E43-9.

9 Thong FSL, Hudson R, Ross R, et al. Plasma leptin in moderately obese men: independent effects of weight loss and aerobic exercise. Am f Physiol Endocrinol Metab 2000;279:E307-13.

10 Kraemer, RR, Kraemer GR, Acevedo EO, et al. Effects of aerobic exercise on serum leptin levels in obese women. Eur f Appl Physiol 1999;80:154-8.

11 Gutin, B, Ramsey L, Barbeau P, et al. Plasma leptin concentrations in obese children: changes during 4-mo periods with and without physical training. Am F Clin Nutr 1999;69:388-94.

\section{A biomechanical perspective: do foot orthoses work?}

Foot orthoses have become an integral part of the treatment of injuries of the foot, ankle, and lower extremity. From a biomechanical perspective, they offer a means of resolving symptoms by placing the foot and the lower extremity in a more advantageous position thus altering applied tissue stresses. Ample evidence exists, based on subjective pain relief and symptom resolution, to support the continued use of these devices. However, scientific evidence to confirm these observations is equivocal.

\section{Research findings}

If there is a biomechanical basis for patient improvement, one of many possible kinematic or kinetic parameters should be altered by foot orthoses. Increased magnitude of the pronation angle and increased pronation velocity have been postulated as risk factors for lower extremity injury. A number of investigations have shown the potential of an orthosis with an external medial post to decrease the magnitude of pronation. ${ }^{1}$ Not unexpectedly, a decrease in tibial internal rotation has also been shown with medially posted orthoses. ${ }^{2}$ However, Johanson et $a l^{1}$ observed that a non-posted orthotic shell reduced the maximum pronation angle as much as either a forefoot or a rearfoot post, as well as a combination of a forefoot and rearfoot post.

However, attempts to reduce the velocity of pronation through foot orthoses have proved less successful. ${ }^{3}$ Pronation velocity may be influenced more than the magnitude of the motion by the eversion moment that results from the point of application of the ground reaction force. Investigations showing reduced motion often find no change in pronation velocity. With a restriction to normal pronation, Perry and Lafortune ${ }^{4}$ found no change in impact loading during walking. However, during running, the same pronation restriction produced an increase in impact loading. This suggests that the influence of an orthosis differs between walking and running and should be considered at the time of prescription.

\section{Research contradictions}

It appears that for every study showing a positive change in a biomechanical parameter produced by foot orthoses, another study can be cited showing no change. Some of these discrepancies could be due to methodological differences. These include the measurement system, marker placement, skin movement artefact, variable subject/ patient groups, lack of statistical power, individual subject response, and the type of orthotic intervention. Reinschmidt et $a \bar{l}$ showed substantial errors between skin markers and intracortical pins in the frontal and transverse planes (63\% and $70 \%$ respectively). Advances in measurement technique should resolve some of the contradictions; however, a recent study using intracortical pins ${ }^{6}$ showed that orthotic effects were subject specific and nonsystematic across conditions.

Orthotic behaviour is generally assessed using some measure of rearfoot motion to describe the subtalar joint action. Unfortunately there is no direct method to do this. Subtalar and talocrural joint motion can only be inferred from the measures that biomechanists often use. Part of the problem may be that the wrong parameters have been measured or that the changes made by the orthoses are too subtle for the measurement system to detect.

In many studies, the subjects are not patients and therefore may not respond to the orthotic intervention as a patient may. The unimpaired subjects may attempt to override any of the "unnecessary" effect of the orthosis that 
would force them into a less efficient locomotory pattern. Nawoczenski et al observed that different foot structures showed different amounts of frontal and transverse plane motion. Both of these factors could contribute to a range of responses in individual subjects, evident by the observation of internal tibial rotation changing from $-80 \%$ to $+60 \%$ with the use of an orthosis. ${ }^{7}$

Further, the foot motion observed may not be dictated primarily by foot structure. Movement patterns of the foot may be driven by (and the effects of orthosis found in) the proximal joints. Bellchamber and van den Bogert ${ }^{8}$ calculated a proximal to distal energy flow between the tibia and foot among all subjects during walking and some subjects during running. From the observed direction of energy flow, the authors suggested that the use of foot orthoses may be ineffective in controlling tibial rotation. This conclusion, however, is countered by studies cited above. $^{7}$

Finally, the variation in patients' response to foot orthoses may be largely influenced by the methods used in fitting. Foot orthoses are typically fitted on the basis of a static clinical examination of various measurements of lower extremity alignment. The assumption is that the position of the foot and ankle in the static position reflects the motion of the foot and ankle during ambulation-that is, an increased static pronation angle will produce an increased maximum pronation angle during ambulation. However, much evidence has shown a rather poor relation between static measures and dynamic lower extremity motion. ${ }^{9}$ Hamill et al ${ }^{9}$ showed that various static clinical measures of lower extremity alignment are limited in predicting dynamic lower extremity function. On the basis of similar results, Hunt et al $l^{10}$ questioned the appropriateness of using such measures in prescribing and fitting foot orthoses. If the static measures do not accurately reflect the dynamic motions of the foot and ankle, then designing an orthosis on the basis of these static measures may not provide adequate correction for the dysfunction. Mueller ${ }^{11}$ suggested that orthoses should not be prescribed on the basis of specific foot alignment measures, but rather on the patient's symptoms.

\section{Summary}

This article is not intended as a comprehensive review of the literature. Rather, its purpose is to bring to the reader's attention several of the key issues involved with foot orthoses and their prescription. In spite of the rather equivocal findings from the numerous investigations involving the efficacy of foot orthoses, their success in reducing pain and symptoms cannot be denied. However, the mechanism by which this is accomplished is certainly open to question. The determination of the mechanism may involve the procedures used to evaluate orthoses biomechanically.

Osteopathic Medical Center

B HEIDERSCHEIT

Des Moines University

Des Moines, Iowa, USA

University of Massachusetts

J HAMILL

jhamill@excsci.umass.edu

D TIBERIO

University of Connecticut

1 Johanson MA, Donatelli R, Wooden MJ, et al. Effects of three different posting methods on controlling abnormal subtalar pronation. Phys Ther ing methods on

2 McPoil TG, Cornwall MW. The effect of foot orthoses on transverse tibial rotation during walking. 7 Am Podiatr Med Assoc 2000;90:2-11.

3 Rodgers MM, Leveau BF. Effectiveness of foot orthotic devices used to modify pronation in runners. F Orthop Sports Phys Ther 1982;4:86-90.

4 Perry SD, Lafortune MA. Influences of inversion/eversion of the foot upon impact loading during locomotion. Clinical Biomechanics 1995;10:253-7.

5 Reinschmidt C, van den Bogert AJ, Nigg BM, et al. Effect of skin movement on the analysis of skeletal knee joint motion during running. F Biomech 1997;30:729-32

6 Stacoff A, Reinschmidt C, Nigg BM, et al. Effects of foot orthoses on skeletal motion during running. Clinical Biomechanics 2000;15:54-64.

7 Nawoczenski DA, Cook TM, Saltzman CL. The effect of foot orthotics on three-dimensional kinematics of the leg and rearfoot during running. $f$ Orthop Sports Phys Ther 1995;21:317-27.

8 Bellchamber TL, van den Bogert AJ. Contributions of proximal and distal moments to axial rotation during walking and running. $\mathcal{F}$ Biomech 2000;33: 1397-403.

9 Hamill J, Bates BT, Knutzen KM, et al. Relationship between selected static and dynamic lower extremity measures. Clinical Biomechanics 1989;4:21725 .

10 Hunt AE, Fahey AJ, Smith RM. Static measures of calcaneal deviation and arch angle as predictors of rearfoot motion during walking. Australian fournal of Physiotherapy 2000;46:9-16.

11 Mueller MJ. Invited commentary: effects of three different posting methods on controlling abnormal subtalar pronation. Phys Ther 1994;74:158-9.

\section{Sports medicine in the Netherlands}

Sports medicine can be defined in different ways. In the Netherlands the definition of sports medicine, the field of work in sports medicine, and training in sports medicine have changed several times since specific sports medical activities began in the 1920s. The Olympic games in Amsterdam (1928) saw the beginning of specific preventive activities in sports medicine. Preseasonal screening was established, and after the second world war more than 300000 preventive preseasonal screenings were performed a year. Another 200000 children were screened annually by school doctors.

In 1965 the Netherlands Association for Sports Medicine was established. Doctors interested in sports medical problems could attend a specific course. The programme was broad and offered general topics ranging from cardiology to orthopaedic surgery and exercise physiology. The character of the course was a retraining course. Its duration was about 40 hours and it formed the basis for membership of the Netherlands Association for Sports Medicine.
Ten years later the first doctor was fully trained in sports medicine partly modelled on East European standards. This education took four years and consisted of one year clinical cardiology, one year clinical orthopaedic surgery, one year exercise physiology in a university exercise laboratory, and one year practical work in the field of sports medicine in places such as the national centre for soccer and the national centre for sport (Olympic centre). Beside these training activities, there was a (general) course in social medicine (12 weeks). For the organisation and quality control of this new discipline, a foundation for training of specialists in sports medicine (Stichting Opleiding Sportartsen; SOS) was established. The SOS had several committees which controlled training content and procedural aspects. Initially, about two doctors started training every year. The specialists in sports medicine set up a separate section of the Netherlands Association for Sports Medicine (1982) and wrote a profile "Fields of activity of specialists in sports medicine" (1983). The aims of training for specialisation in sports medicine were formulated on 
the basis of this document (1985), which formed the basis for the recognition of sports medicine as an official discipline of social medicine by the College of Social Medicine. A year later (1987) the degree was given legal power by the secretary of state for health, welfare and cultural affairs. During this period, the inflow of professionals increased to six to eight persons a year, and a new curriculum was formulated. In 1991 the curriculum for the training of specialists in sports medicine was published (77 pages, also published in English).

Registration or recognition in one of the three fields of medicine in the Netherlands is important in many ways. Doctors in the Netherlands can be divided into three groups: general practitioners, social medicine experts, and specialists. Another division could be made: preventive medicine and curative medicine with subdivision into extramural and intramural. The three kinds of health care and the three groups of doctors are paid from different sources. Each group has to decide what kind of medicine it covers. After acceptance, the ministry of health normally takes the advice of the board of specialists (Central College) and the College of Social Medicine.

In 1993 the first sports doctor started a full time job in a hospital. The field of sports medicine, described in 1982 by the section of specialists in sports medicine of the Netherlands Association for Sports Medicine, was changed by this initiative. The work was much more therapeutic than previously, and the importance of diagnosis in everyday work was completely different from before 1993. A new discussion about the definition of (the field of) sports medicine was begun. Now, seven years later, about 20 hospitals have a sports medical department (or intend to have one in the near future) run by a professional sports physician. Three university hospitals belong to this group. In the first hospital, about 1000 patients with sports related medical prob- lems visit the department a year sent by their general practitioner or another specialist working in the same hospital. Most of the patients are ordinary athletes not involved in high level training and competition. Along with the patient related activity, there is still preventive screening for specific sports. In addition to this obligatory screening, some athletes and even non-athletes ask to be screened. The third activity is the care provided for top level athletes. This health care is often given outside of a hospital. All the sports medical departments in a hospital have a well equipped exercise physiology laboratory partly for diagnosis and partly for the control of and training advice for top athletes.

The change from working outside the clinic before 1993 to inside the clinic after was combined with a request for sports doctors to be registered as specialists. One of the most important reasons for this request is that financial support for hospital care is based on registration of the doctor. For a specialist, the financial support for health care in a hospital is much greater than for a doctor registered in social medicine. The request to be specialists was presented to the board of specialists last year and is still very much the topic of medical and political debate. If the request is accepted, training in sports medicine must be changed again in line with other specialist training. The new field of sports medicine will need to be defined again, and a political debate is underway to chart the field and state of science in sports medicine. A new decade in sports medicine in the Netherlands seems to be on the way.

G C VAN ENST

Sports physician, Isala Clinics, loc sophia

Sports Medicine Department, pb 10400

8000 GK Zwolle, the Netherlands

gvanenst@wxs.nl

\section{Toxocara: dogwalking and playing fields}

In villages, towns, and cities throughout the country, land is set aside for human recreation. Public and private playing fields enable the population to indulge in sport and recreation, but these areas are often convenient for another form of human activity: dog walking. Sportsmen may be concerned that toxocaral infection may be acquired from canine defaecation on public playing fields, and the fastidious groundsmen may remove offending articles before matches are played. However, in doing so, they do little to reduce the risk of toxocaral infection.

\section{Life cycle}

Toxocara canis is the round worm (ascarid) parasite of canids: dogs and foxes. It has a complex and unusual life cycle. Eggs, when ingested, hatch in the small intestine and invade the intestinal wall. They are taken up in the portal system and distributed through the liver-lung migration cycle, going through larval moults, and being coughed up and swallowed when they develop into adult worms. This characteristic ascarid life cycle is confined mainly to dogs under the age of six months; above this age, larval development is arrested at the second larval stage (L2). This apparent dead end is important for the survival of the parasite as these L2 larvae are re-activated during pregnancy and migrate across the placenta to invade the puppies, and larvae are also excreted in the milk. Consequently, almost all puppies develop an active toxocaral infection. Eggs are also infective for a wide range of mammalian hosts, but larvae are unable to develop beyond the L2 stage and continue to migrate through the body. ${ }^{1}$ Toxocara larvae excrete a complex mixture of glycoproteins that are potent stimulators of the host immune system, and it is this that is responsible for the characteristic symptoms and signs of toxocaral infection. ${ }^{2}$

\section{Human disease}

Toxocariasis takes three main forms: an occult form characterised by failure to thrive and abdominal pains in children and visceral larva migrans characterised by fever, wheeze, cough, and eosinophilia. ${ }^{13}$ In addition, there is an ocular form when trapped larvae stimulate a potent immune response leading to a spectrum of problems including endophthalmitis, uveitis, pars planitis, and subsequent granuloma formation. ${ }^{4}$ Ocular toxocariasis is relatively uncommon: some estimates suggest between 50 and 100 new cases of ocular disease a year in the British Isles. In contrast, asymptomatic toxocariasis is common, with $5-7 \%$ of an adult population in industrialised countries having evidence of previous infection. ${ }^{1}$

So what are the risks to sportsmen on the playing fields? Firstly, fresh Toxocara infected faeces pose no threat to human health because eggs must embryonate, a process that takes up to one month, and only $6-15 \%$ of dogs excrete eggs. ${ }^{1}$ The consequences of this are detected in 
surveys of parks within cities in the United Kingdom, which indicate that between 5 and $10 \%$ of samples will have infective Toxocara eggs. ${ }^{5}$ Visceral larva migrans peaks in children aged three years and is more common in boys. ${ }^{3}$ Ocular disease presents slightly later in life, between 7 and 10 years. ${ }^{4}$ Adults appear to be relatively resistant to symptomatic infection-for example, surveys of hydatid control in New Zealanders who have intense contact with dogs show that more than $25 \%$ have antibodies to Toxocara without any evidence of clinical disease. ${ }^{6}$ Thus one would expect the risk for adult sportsmen from contaminated fields to be relatively low, but the risk for children is higher but hard to quantify.

In summary, promiscuous canine defaecation poses a significant aesthetic hazard in playing fields. For children, there is a risk of serious toxocaral infection that may lead to visceral larva migrans or visual loss. For adults, the risk is much lower. Therefore everyone involved in the management of playing fields, whether public or private, should encourage dog owners to be responsible, clear up after their pets, and take steps to enforce this behaviour.

S GILLESPIE

Department of Medical Microbiology

Royal Free and University College London

Royal Free Campus

London NW3 2PF, UK

stepheng@rfc.ucl.ac.uk

1 Gillespie SH. The epidemiology of Toxocara canis. Parasitology Today 1998;4:180-2

2 Gillespie SH. The clinical spectrum of human toxocariasis. In: Toxocara and toxocariasis. Clinical, epidemiological and molecular perspectives. London: Institoxocariasis. Clinical, ep
tute of Biology, 1993.

3 Glickman LT, Schantz PM. Epidemiology and pathogenesis of toxocariasis. Epidemiol Rev 1981;3:230-50

4 Gillespie SH, Dinning WJ, Voller A, Crowcroft NS. The spectrum of ocular toxocariasis. Eye 1993; 7:415-18.

5 Gillespie SH, Pereira M, Ramsay A. The prevalence of Toxocara canis in soil samples from parks and gardens in the London area. Public Health 1991;105:335-9.

6 Clemett RS, Hidajat RR, Allardyce RA. Toxocaral infection in hydatid control officers: diagnosis by immunoassay. $N Z$ Med $\mathcal{F}$ 1985;98:737-9. 